\title{
Actinomicetos produtores de inibidores de $\beta$-lactamases com atividade antimicrobiana frente a isolados de mastite bovina
}

\author{
[Actinomycetes producers of $\beta$-lactamases inhibitors with antimicrobial activity against microorganisms \\ isolated from bovine mastitis]
}

\author{
M.N.C. Cunha ${ }^{1,2}$, N.M.V. Silva ${ }^{3}$, M.F.S. Teixeira ${ }^{3}$, R.A. Mota ${ }^{4}$, J.L. Lima-Filho ${ }^{2}$, \\ T.S. Porto ${ }^{2,4}$, A.L.F. Porto ${ }^{2,4^{*}}$ \\ ${ }^{1}$ Aluna de pós-graduação - Biociência Animal - URFPE - Recife, PE \\ ${ }^{2}$ Laboratório de Imunopatologia Keizo Asami - UFPE - Recife, PE \\ ${ }^{3}$ Departamento de Parasitologia - UFAM - Manaus, AM \\ ${ }^{4}$ Universidade Federal Rural de Pernambuco - Recife, PE
}

\begin{abstract}
RESUMO
Avaliou-se a capacidade de 71 actinomicetos isolados de líquens da região amazônica em produzir inibidores de $\beta$-lactamases com atividade antimicrobiana sobre Staphylococcus aureus, resistentes à penicilina, isolados de mastite bovina do estado de Pernambuco. A seleção dos actinomicetos produtores de inibidores de $\beta$-lactamases foi realizada pela técnica de bloco de gelose contra Klebsiella pneumoniae ATCC 29665, e os actinomicetos selecionados foram testados frente a 17 linhagens de Staphylococcus aureus resistentes à penicilina. Os melhores produtores de inibidores de $\beta$-lactamases foram Streptomyces sp. DPUA 1542 e Nocardia sp. DPUA 1571, os quais foram submetidos ao cultivo submerso para determinação da curva de crescimento, $\mathrm{pH}$ e atividade antimicrobiana. Os maiores halos de inibição foram obtidos pelos metabólitos produzidos após 96 horas de cultivo tanto para Nocardia sp. - 13,5 e 12,0mm - como para Streptomyces sp. - 8,0 e 14,0mm - com os testes de difusão nos discos e poços, respectivamente. Os resultados permitiram concluir que os actinomicetos são fonte promissora de inibidores de $\beta$-lactamases, com potencial uso no tratamento de mastites bovinas.
\end{abstract}

Palavras-chaves: mastite bovina, inibidor de $\beta$-lactamase, actinomiceto, atividade antimicrobiana, Staphylococcus aureus

\begin{abstract}
The ability of 71 actinomycetes, isolated from the Amazon lichens, to produce $\beta$-lactamase inhibitors with antimicrobial activity was evaluated against penicillin-resistant Staphylococcus aureus, isolated from bovine mastitis in Pernambuco State. The selection of actinomycetes producers of $\beta$-lactamase inhibitors was performed using agar-plug method against Klebsiella pneumoniae ATCC 29665 and the selected actinomycetes were tested against 17 penicillin-resistant Staphylococcus aureus strains. The best producers of $\beta$-lactamase inhibitors were Streptomyces sp. DPUA 1542 and Nocardia sp. DPUA 1571. They were submitted to the submerged cultivation to determine the growth and $\mathrm{pH}$ curve, and antimicrobial activity. The highest inhibition halo zone was obtained by metabolites produced after 96 hours of cultivation for both Nocardia sp. (13.5 and 12.0mm) and Streptomyces sp. (8.0 and $14.0 \mathrm{~mm}$ ) with discs and well diffusion tests, respectively. The results showed that the actinomycetes are a promising source of $\beta$-lactamase inhibitors, with potential for use in the bovine mastitis treatment.
\end{abstract}

Keywords: bovine mastitis, $\beta$-lactamase inhibitor, actinomycetes, antimicrobial activity, Staphylococcus aureus

Recebido em 7 de abril de 2010

Aceito em 27 de dezembro de 2010

*Autor para correspondência (corresponding author)

E-mail: analuporto@yahoo.com.br 


\section{INTRODUÇÃo}

A mastite é a inflamação da glândula mamária de diferentes etiologias e em $90 \%$ dos casos é causada por bactérias (Benedette et al., 2008; Tozzeti et al., 2008). A mastite bovina é apontada como a principal doença dos rebanhos leiteiros no mundo inteiro, causando prejuízos econômicos tanto ao produtor de leite quanto à indústria de laticínios (Tozzeti et al., 2008), pela redução da quantidade e pelo comprometimento da qualidade do leite produzido, ou até pela perda total da capacidade secretora da glândula mamária (Ribeiro et al., 2003).

Staphylococcus aureus é reconhecido como o principal patógeno nos casos de mastite (Salasia, 2004). Ainda que o controle da mastite fundamente-se principalmente nas medidas higiênico-sanitárias, a antibioticoterapia exerce papel importante no caso de infecções por bactérias, tendo em vista a possibilidade de eliminar as infecções intramamárias e reduzir prováveis fontes de infecção (Erskine et al., 1993). Um dos fatores que vem sendo considerado para o controle das mastites é a resistência dos agentes etiológicos aos antimicrobianos (Zafalon et al., 2008).

O uso indiscriminado e prolongado de antibióticos tem levado à seleção de microrganismos patogênicos resistentes a esses compostos (Tresoldi et al., 2000). Um dos mecanismos mais importantes da resistência exibidos por uma variedade de bactérias Grampositivas e Gram-negativas é sua habilidade em produzir $\beta$-lactamases. Essas enzimas inativam penicilinas e cefalosporinas por meio da hidrólise do anel $\beta$-lactâmico (Brown et al., 1976). O grau de resistência da bactéria depende da quantidade de enzima produzida, da habilidade dessa enzima em hidrolisar o antimicrobiano em questão e da velocidade com que o $\beta$-lactâmico penetra pela membrana externa da bactéria (Macedo et al., 2005).

Estratégias para inativar essas enzimas têm assumido importância crítica na área médica (Bethel et al., 2004). Uma proposta para contornar esse problema é a utilização de um inibidor de $\beta$-lactamase juntamente com um antibiótico $\beta$-lactâmico (Baptista Neto, 2004). Os inibidores de $\beta$-lactamases são compostos similares aos antibióticos, que se ligam 稀 - lactamases de forma geralmente irreversível, protegendo os antibióticos contra sua destruição, garantindo sua atividade frente a microrganismos patogênicos (Macedo et al., 2005).

Os objetivos deste trabalho foram testar a produção de $\beta$-lactamase e verificar a atividade antimicrobiana sobre $S$. aureus resistentes à penicilina e à amoxicilina.

\section{MATERIAL E MÉTODOS}

Os microrganismos utilizados foram 71 actinomicetos isolados de líquens da região amazônica produtores de inibidores de $\beta$ lactamase, pertencentes à coleção de microrganismos do Departamento de Parasitologia da Universidade do Amazonas (DPUA), cedidos pela Profa. Maria Francisca Simas Teixeira.

Os microrganismos-teste utilizados foram 17 linhagens de $S$. aureus isolados de mastite bovina de rebanhos do estado de Pernambuco, resistentes à penicilina (Medeiros et al., 2009), cedidas pelo Prof. Rinaldo Aparecido Mota, do Departamento de Medicina Veterinária da UFRPE, e K. pneumoniae ATCC 29665 produtora de $\beta$-lactamase.

O meio de cultura utilizado para manutenção das amostras de actinomicetos foi o meio ISP-2 (Pridham et al., 1957), modificado pela retirada de glicose. As culturas de actinomicetos foram semeadas em placas de Petri contendo o meio ISP-2, incubadas a $30^{\circ} \mathrm{C}$ por 168 horas e mantidas em temperatura ambiente. As culturas foram repicadas a cada 30 dias. $\mathrm{O}$ caldo nutriente foi utilizado como o meio de manutenção para os microrganismos-teste $K$. pneumoniae ATCC 29665 e S. aureus. Todos os microrganismosteste foram conservados a $-20^{\circ} \mathrm{C}$ como cultura estoque em criotubos, utilizando-se glicerol $20 \%$ (v/v).

A produção de inibidores $\boldsymbol{\beta}$ e -lactamases foi avaliada por meio da técnica de bloco de gelose por difusão em ágar, segundo Brown et al. (1976), utilizando-se $K$. pneumoniae ATCC 29665 como microrganismo teste. Os testes foram realizados em ágar Müeller-Hinton adicionado de solução de benzil-penicilina $G$ $(0,01 \mu \mathrm{g} / \mathrm{mL})$. O meio foi distrib́do em placas de Petri (90x15mm), e na superfície do meio foi 
semeada uma suspensão do microrganismo-teste. Os microrganismos-teste foram cultivados em Erlenmeyers (50mL) contendo o meio Tryptic Soy Broth (TSB), a $37^{\circ} \mathrm{C}$ por 24 horas, para atingir a concentração de $10^{8} \mathrm{UFC} / \mathrm{mL}$, o que correspondeu a uma solução padrão de McFarland a 0,5, ou seja, um valor de absorbância igual a 0,1 a 600nm, segundo National Comite Clinical Laboratory Standards (NCCLS) (Methods..., 2003). Nas placas foram adicionados fragmentos de $6 \mathrm{~mm}$ de diâmetro, retirados da área central das culturas dos actinomicetos, cultivados em meio ISP-2 sólido a $30^{\circ} \mathrm{C}$, por 168 horas. $\mathrm{O}$ controle utilizado foi o clavulanato de potássio (Sigma Aldrich - São Paulo, Brasil), na concentração de $10 \mathrm{mg} / \mathrm{mL}$. Após a incubação das placas a $37^{\circ} \mathrm{C}$ por 24 e 48 horas, foram realizadas leituras dos diâmetros dos halos de inibição, expressos em milímetros.

A técnica utilizada nesta etapa foi a de bloco de gelose por difusão em ágar, segundo Brown et al. (1976), como descrito no item anterior. Os microrganismos testados foram 17 estirpes de $S$. aureus isoladas de mastite bovina e resistentes à penicilina e à amoxilina.

Fragmentos de 6mm de diâmetro, retirados da área central das colônias dos actinomicetos, crescidos em meio ISP-2 a $30^{\circ} \mathrm{C}$ por 168 horas, foram cultivados em Erlenmeyer $(125 \mathrm{~mL})$ contendo $25 \mathrm{~mL}$ do meio ISP-2 e mantidos sob agitação (200rpm) a $28^{\circ} \mathrm{C}$ por 48 horas. Após esse período, $10 \%(\mathrm{v} / \mathrm{v})$ deste pré-inóculo foi transferido para Erlenmeyer de $250 \mathrm{~mL}$ contendo $100 \mathrm{~mL}$ do meio de cultivo MS-2 descrito por Porto et al. (1996). Os cultivos foram realizados em agitador orbital (200rpm) a $28^{\circ} \mathrm{C}$, por um período de 144 horas, e a cada 24 horas um Erlenmyer era retirado para acompanhar a cinética de crescimento, a curva de $\mathrm{pH}$ e a determinação da atividade antimicrobiana do líquido metabólico.

A atividade dos inibidores de $\beta$-lactamases nos líquidos metabólicos produzidos pelos actinomicetos foi estimada empregando-se o método biológico de difusão em disco, descrito por Ericsson e Sherris (1971), e a de difusão em poço. Na técnica de difusão em disco, placas contendo ágar Müeller Hinton, adicionado com uma solução de benzil-penicilina $\mathrm{G}(0,01 \mu \mathrm{g} / \mathrm{mL})$, foram semeadas com uma suspensão dos microrganismos-teste $K$. pneumoniae ATCC 29665 e $S$. aureus isolados de mastite bovina, cultivados em meio TSB a $37^{\circ} \mathrm{C}$ por 24 horas, para atingir a concentração de $10^{8}$ UFC, que corresponde a uma solução padrão de McFarland (Methods..., 2003). Nestas placas foram adicionados discos de papel de filtro de $6 \mathrm{~mm}$ de diâmetro, impregnados com $10 \mu \mathrm{L}$ dośquidos metabólicos livres de células, $\mathrm{E}$ as placas foram incubadas a $37^{\circ} \mathrm{C}$ por 24 horas.

Para o teste de difusão em poço, o mesmo meio de cultura foi utilizado e distribuído em placas de Petri, onde foi semeada uma suspensão dos microrganismos-teste na mesma concentração utilizada no teste de difusão em disco. No centro das placas de Petri, foram realizados orifícios de $6 \mathrm{~mm}$ de diâmetro, onde foram adicionados $30 \mu \mathrm{L}$ do líquido metabólico produzido pelos actinomicetos selecionados. As placas foram incubadas em estufa a $37^{\circ} \mathrm{C}$ por 24 horas. Em seguida, foram realizadas as leituras dos diâmetros dos halos de inibição expressos em $\mathrm{mm}$. Os ensaios foram realizados em duplicata.

A avaliação da biomassa foi realizada por gravimetria (peso seco), e o $\mathrm{pH}$ foi medido por potenciometria no decorrer do cultivo a cada 24 horas.

\section{RESULTADOS E DISCUSSÃO}

Os ensaios em bloco de gelose mostraram que, dentre as 71 amostras de actinomicetos testadas, 13 (18\%) apresentaram atividade antimicrobiana frente ao microrganismo-teste, $K$. pneumoniae ATCC 29665, produtor de $\beta$-lactamase após 24 horas de cultivo, e 15 (22\%) após 48 horas de cultivo. As melhores atividades antimicrobianas obtidas frente a K. pneumoniae ATCC 29665 após 24 horas de cultivo foram apresentadas pelas amostras de Streptomyces sp. DPUA 1542 e DPUA 1543, e por Nocardia sp. DPUA 1571, com halos de inibição de $30 \mathrm{~mm}$, 28mm e $24 \mathrm{~mm}$, respectivamente (Tab. 1). Ceylan et al. (2008) isolaram 15 amostras de Streptomyces sp. do solo da Turquia, estudaram suas atividades antimicrobianas frente a S. aureus, Escherichia coli, Pseudomonas aeruginosa e Stenotrophomonas maltophilia e obtiveram halos de inibição de $\geq 30 \mathrm{~mm}$. Os resultados desses autores foram confirmados no presente trabalho. 
Actinomicetos produtores de inibidores...

Tabela 1. Atividade antimicrobiana expressa por halos de inibição produzidos pelos actinomicetos frente a K.pneumoniae para seleção dos produtores de inibidores ße -lactamases pela técnica do bloco de gelose

\begin{tabular}{lcc} 
Amostra & $\begin{array}{c}\text { Diâmetro do halo de inibição } \\
(\mathrm{mm})\end{array}$ & $\begin{array}{c}\text { Diâmés 24 horas } \\
(\mathrm{mm}) \text { após 48 horas }\end{array}$ \\
\hline Streptomyces sp. DPUA 1542 & $\mathbf{3 0 , 0}$ & $\mathbf{3 9 , 0}$ \\
Streptomyces sp. DPUA 1543 & $\mathbf{2 8 , 0}$ & $\mathbf{3 7 , 0}$ \\
Streptomyces sp. DPUA 1547 & 10,0 & 10,0 \\
Streptomyces sp. DPUA 1549 & 14,0 & 17,0 \\
Streptomyces sp. DPUA 1550 & 18,0 & 18,0 \\
Streptomyces sp. DPUA 1557 & 14,0 & 14,0 \\
Streptomyces sp. DPUA 1559 & 10,0 & 14,0 \\
Streptomyces sp. DPUA 1566 & 10,0 & 10,0 \\
Streptomyces sp. DPUA 1568 & 16,0 & 16,0 \\
Nocardia sp. DPUA 1571 & $\mathbf{2 4 , 0}$ & $\mathbf{3 8 , 0}$ \\
Streptomyces sp. DPUA 1573 & 18,0 & 24,0 \\
Nocardia sp. DPUA 1577 & 14,0 & 14,0 \\
Streptomyces sp. DPUA 1586 & - & 14,0 \\
Streptomyces sp. DPUA 1587 & - & 12,0 \\
Streptomyces sp. DPUA 1591 & 14,0 & 14,0 \\
Streptomyces sp. DPUA 1605 & - & 10,0 \\
\hline
\end{tabular}

Os actinomicetos selecionados, os quais apresentaram maior atividade antimicrobiana frente ao microrganismo-teste $K$. pneumoniae ATCC 29665, foram submetidos novamente a testes de bloco de gelose frente a 17 linhagens de $S$. aureus isolados de mastite bovina e resistentes à penicilina. Os resultados da atividade antimicrobiana das linhagens de actinomicetos frente aos isolados de mastite bovina estão apresentados na Tab. 2.

Das 16 amostras de actinomicetos préselecionadas, apenas Streptomyces sp. DPUA 1547 e Streptomyces sp. DPUA 1605 não apresentaram atividade antimicrobiana frente aos isolados de mastite bovina. Os actinomicetos Streptomyces sp. DPUA 1542 e Nocardia sp. DPUA 1571 foram os que apresentaram maiores halos de inibição frente a $S$. aureus, 28 e 31mm, respectivamente (Tab. 2). Estes resultados comprovam a produção de inibidores de $\beta$ lactamases, pois os $S$. aureus utilizados neste estudo foram caracterizados por Medeiros et al. (2009), que estudaram o perfil de sensibilidade microbiana in vitro de 291 linhagens de Staphylococcus spp. isoladas de mastite bovina, e foram testadas frente aos seguintes antibióticos: amoxicilina, ampicilina, azitromicina, cefquinome, cephalonium, ciprofloxacina, cloxacilina, danofloxacina, enrofloxacina, eritromicina, florfenicol, gentamicina, penicilina + novobiocina, trimetoprim, tobramicina, tetraciclina+ neomicina+ bacitracina. Os autores observaram também um perfil de multirresistência para 65 dos $S$. aureus isolados, em que: 44 estirpes apresentaram resistência de 2 - 4, 17 estirpes resistência de 5 - 7, e quatro foram resistentes a oito ou mais antibióticos.

Al-Zahrami (2007), ao estudar a atividade antimicrobiana de Streptomyces sp. isolado do solo da Arábia Saudita, frente a amostras de $S$. aureus, pela técnica bloco de gelose e utilizando blocos de $5 \mathrm{~mm}$ de diâmetro, observou halos de inibição de até $29 \mathrm{~mm}$ de diâmetro, resultado semelhante aos obtidos neste estudo. Thakur et al. (2007) isolaram 110 amostras de actinomicetos do solo da Índia e destas, 39 (60\%) apresentaram atividade antimicrobiana frente a $S$. aureus. Das amostras testadas neste estudo contra estirpes de $S$. aureus, 14 apresentaram atividade inibitória, o que corresponde a $87,5 \%$ do total. 


\section{Cunha et al.}

Tabela 2. Atividade antimicrobiana expressa por halos de inibição $(\mathrm{mm})$ apresentados pelos actinomicetos frente às estirpes de Staphylococcus aureus isolados de mastite bovina pela técnica do bloco de gelose

\begin{tabular}{|c|c|c|c|c|c|c|c|c|c|c|c|c|c|c|c|c|}
\hline & 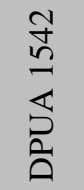 & 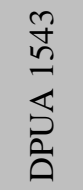 & 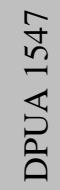 & 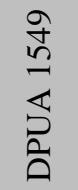 & 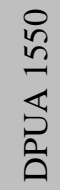 & 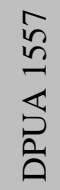 & 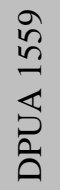 & 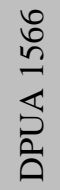 & 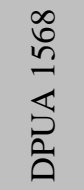 & 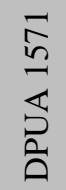 & 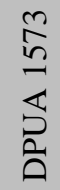 & 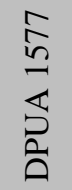 & 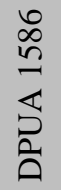 & 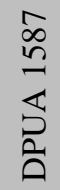 & 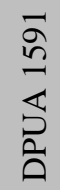 & 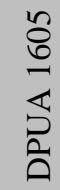 \\
\hline S1 & - & - & - & - & - & - & - & - & - & - & - & - & - & - & - & - \\
\hline S2 & 13 & 11 & - & 8 & 8 & - & 9 & 8 & 10 & 12 & - & 8 & 8 & - & 8 & - \\
\hline S3 & 28 & 18,5 & - & 10 & 8 & - & - & 13 & - & 23 & 13 & - & - & - & 11 & - \\
\hline S4 & - & - & - & - & - & - & - & - & - & - & - & - & - & - & - & - \\
\hline S5 & 26 & 19 & - & 9 & 9 & - & 8 & 9 & 19 & 21 & 10 & - & - & - & 15 & - \\
\hline S6 & 20 & 14 & - & 12 & - & 13 & 7 & 9 & 12 & 24 & 7 & 7 & - & - & 12 & \\
\hline S7 & 19 & 12,5 & - & - & - & - & - & - & - & 19 & - & - & - & - & - & - \\
\hline S8 & 24 & 18,5 & - & 10 & 8 & - & - & 9 & 13,5 & 23 & 13 & 7,5 & - & - & 10 & - \\
\hline S9 & 28 & 29 & - & 18,5 & 13 & - & 19 & 12 & 18 & 28 & 8 & 10 & - & - & 15 & - \\
\hline S10 & 24 & 21 & - & 14 & - & - & - & 11 & - & 30 & 10 & - & - & - & 10 & - \\
\hline S11 & 26,5 & 21 & - & 15 & 18 & - & - & 9 & 11 & 31 & 9 & 8 & - & - & 10 & - \\
\hline S12 & 24,5 & 19 & - & 13,5 & 13 & - & - & 13 & 19 & 21 & 8 & 7 & - & - & 12 & - \\
\hline S13 & 24,5 & 19 & - & 11,5 & 15 & - & - & 12 & 14 & 28 & 11 & 8 & - & - & 12 & - \\
\hline S14 & 25 & 16 & - & 11 & - & - & - & - & 13,5 & 26 & 12 & 12 & - & - & - & - \\
\hline S15 & 19 & 13 & - & 17 & 12 & - & - & 10 & 13 & 30 & 8 & 7 & - & 7,5 & 13 & - \\
\hline S16 & 27 & 19 & - & 17 & 12 & - & - & 10 & 13 & 30 & 8 & 7 & - & 7,5 & 13 & - \\
\hline S17 & - & - & - & - & - & - & - & - & - & - & - & - & - & - & - & - \\
\hline
\end{tabular}

Streptomyces sp. DPUA 1542 e Nocardia sp. DPUA 1571 apresentaram um perfil de crescimento característico durante o cultivo em meio líquido (MS-2), como apresentado na Fig. 1. Durante o crescimento microbiano, os microrganismos apresentaram uma fase lag de 24 horas e uma fase logarítmica característica, a qual exibiu crescimento máximo no tempo 72 horas para Nocardia sp. DPUA 1571 e 96 horas para Streptomyces sp. DPUA 1542. Este apresentou maior valor de biomassa do que Nocardia sp. DPUA 1571, nas mesmas condições de cultivo (Fig. 1). O pH do meio de cultivo aumentou de 7,0 para 8,5 ao final das 144 horas de cultivo (Fig. 1).

Verificou-se, tanto para Streptomyces sp. DPUA 1542 como para Nocardia sp. DPUA 1571, que a produção de metabólitos com atividade antimicrobiana teve início no tempo correspondente ao início da fase estacionária do crescimento microbiano, ou seja, como metabólito secundário. Estes resultados assemelham-se aos descritos por $\mathrm{Yu}$ et al. (1999), que descreveram que a produção de metabólitos secundários pelos actinomicetos em meio líquido é limitada à fase estacionária, que frequentemente coincide com a escassez de nutrientes no meio de cultura.

Durante o crescimento também foi avaliada a atividade antimicrobiana do líquido metabólico produzido pelas culturas dos actinomicetos, por meio da técnica de difusão em discos e em poços, e os resultados estão apresentados na Tab. 3. Foi possível observar a atividade antimicrobiana apresentada pelo Streptomyces sp. DPUA 1542 e Nocardia sp. DPUA 1571 com diâmetros de halos de inibição de 8,00 e 13,5mm utilizando $S$. aureus e de 9,00 e $12,00 \mathrm{~mm}$ com $K$. pneumoniae, respectivamente. Streptomyces sp. DPUA 1542 produziu halos de inibição apenas no tempo de 96 horas de crescimento, já Nocardia sp. DPUA 1571 produziu halos de inibição em 72 horas e 96 horas de crescimento, respectivamente. 


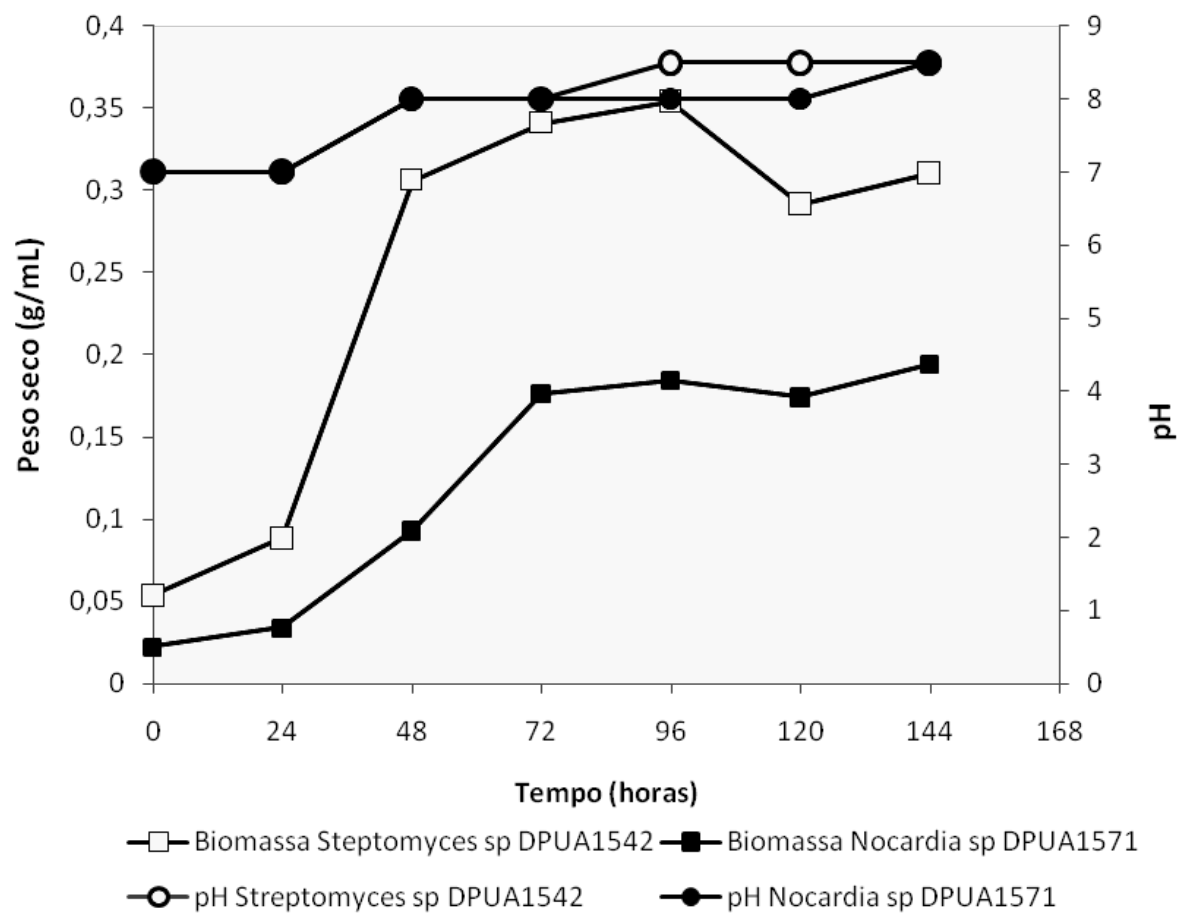

Figura 1. Curva de crescimento e de pH de Streptomyces sp. DPUA 1542 e de Nocardia sp. DPUA 1571 cultivados no meio MS-2 em agitador orbital (200rpm) a $28^{\circ} \mathrm{C}$ por 144 horas.

Tabela 3. Atividades antimicrobianas apresentadas por Streptomyces sp. (DPUA 1542) e Nocardia sp. (DPUA 1571), expressas por halos de inibição (em $\mathrm{mm}$ ) frente aos microrganismos-teste Klebsiella pneumoniae e Staphylococcus aureus isolados de mastite bovina pela técnica de difusão em disco e poço

\begin{tabular}{|c|c|c|c|c|c|c|c|c|}
\hline \multirow{2}{*}{ Microrganismo } & \multicolumn{4}{|c|}{ Streptomyces sp. DPUA 1542} & \multicolumn{4}{|c|}{ Nocardia sp. DPUA 1571} \\
\hline & $48 \mathrm{~h}$ & $72 \mathrm{~h}$ & $96 h$ & $120 \mathrm{~h}$ & $48 \mathrm{~h}$ & $72 \mathrm{~h}$ & $96 \mathrm{~h}$ & $120 \mathrm{~h}$ \\
\hline $\begin{array}{l}\text { K. pneumoniae } \\
\text { (Disco) }\end{array}$ & - & - & 9,00 & - & - & 12,00 & 12,00 & - \\
\hline $\begin{array}{l}\text { K. pneumoniae } \\
\text { (Poço) }\end{array}$ & - & 12,25 & 14,25 & 10,00 & - & 12,50 & 17,50 & 9,00 \\
\hline $\begin{array}{l}\text { S. aureus } \\
\text { (Disco) }\end{array}$ & - & - & 8,00 & - & - & 9,00 & 13,50 & - \\
\hline $\begin{array}{l}\text { S. aureus } \\
\text { (Poço) }\end{array}$ & - & 11,00 & 10,50 & 9,5 & - & 11,00 & 9,00 & 12,00 \\
\hline
\end{tabular}

Para a atividade antimicrobiana que utilizou a técnica difusão em poço, foi possível detectar halos de inibição na maioria dos tempos para os dois microrganismos. Esta maior detecção ocorreu devido à maior quantidade de líquido metabólico $(30 \mu \mathrm{L})$, bem como pela sua maior difusão, utilizando-se esta metodologia (Tab. 3).

De acordo com o NCCLS (Methods..., 2003), um microrganismo é sensível à combinação $\beta$ lactâmico/inibidor de $\beta$-lactamase quando, ao utilizar de 20 a $10 \mu \mathrm{g}$ desse composto em testes de difusão em disco, produz halos $\geq 18 \mathrm{~mm}$ de diâmetro de inibição. Na comparação dos halos de inibição, apresentados por Streptomyces sp. DPUA 1542 e por Nocardia sp. DPUA 1571 frente aos microrganismos-teste $K$. pneumoniae ATCC 29665 e $S$. aureus resistentes à penicilina, não foram obtidos halos maiores que $18 \mathrm{~mm}$. No entanto, as amostras testadas não podem ser classificadas como resistentes, visto que a concentração de inibidores $\beta$ de -lactamases contida nos líquidos metabólicos não foi determinada e, como se encontram em solução, supõe-se que as concentrações são menores que as recomendadas pelas normas da NCCLS. 
Os resultados obtidos neste trabalho corroboram com os dados apresentados por Alberton et al. (2006), que estudaram a atividade antimicrobiana do líquido metabólico obtido da fermentação do Streptomyces viridosporus T7A e observaram que este não foi capaz de inibir o crescimento de Salmonela sp., Pseudomonas sp. e Escherichia coli. O extrato bruto também não promoveu grandes halos de inibição frente às culturas de $S$. aureus causadoras de intoxicação alimentar e de mastites. Esses autores justificaram os resultados obtidos em razão de o metabólito estar diluído no extrato bruto, por isso a utilização de um processo de separação e purificação, visando concentrar esse extrato, irá possibilitar melhores resultados.

\section{CONCLUSÕES}

Estes resultados permitiram concluir que os actinomicetos Streptomyces sp. DPUA 1542 e Nocardia sp. DPUA 1571 foram capazes de produzir inibidores de $\beta$-lactamases, quando testados frente a amostras de $S$. aureus resistentes à penicilina, isoladas de mastite bovina. Avaliações futuras deverão ser realizadas visando otimizar a produção e identificação desse metabólito para aplicação no tratamento de mastites bovinas.

\section{AGRADECIMENTOS}

Os autores agradecem o apoio financeiro do CNPQ e as bolsas de estudo da CAPES e da FACEPE.

\section{REFERÊNCIAS BIBLIOGRÁFICAS}

ALBERTON, L.R.; VANDENBERGHE, L.P.S.; JOINEAU, M.E. et al. Avaliação do potencial de uso do extrato bruto da fermentação por Streptomyces vidosporium T7A em medicina veterinária. Arq. Cien. Vet. Zool. UNIPAR, v.9, p.41-47, 2006.

AL-ZAHRAMI, S.H.M. Studies on antimicrobial activity of Streptomyces sp. isolated from Jazan. JKAU: Sci., v.19, p.127-138, 2007.

BAPTISTA NETO, A. Estudo do processo de produção de ácido clavulânico por Streptomyces clavuligerus em cultivos contínuos com alta concentração celular. 2004. 206f. Tese (Doutorado) - Universidade de São Carlos, São Carlos, SP.
BENEDETTE, M.F.; SILVA, D.; ROCHA, F.P.C. et al. Mastite bovina. Rev. Cien. Elet. Med. Vet., ed.11, 2008.

BETHEL C.R.; HUJER A.M.; HELFAND M.S. et al. Exploring the effectiveness of tazobactam against ceftazidime resistant Escherichia coli: insights from the comparison between susceptibility testing and b-lactamase inhibition. FEMS Microbiol. Lett., v.234, p.99-103, 2004.

BROWN, A.G.; BUTTERWORTH, D.; COLE, $M$. et al. Naturally occurring $\beta$-Lactam inhibitors with antibacterial activity. J. Antibiot., v.29, p.668-669, 1976.

CEYLAN, O.; OKMEN, G.; UGUR, A. Isolation of soil Streptomyces as source antibiotics active against antibiotic-resistant bacteria. EurAsia J. BioSci., v.2, p.73-83, 2008.

ERICSSON, H.M.; SHERRIS, J.C. Antibiotic sensitivity testing-Report of an International Collaborative Study. Acta Pathol. Microbiol. Scand B Microbiol. Immunol., suppl.217, p.1-90, 1971.

ERSKINE, R.J.; KIRK, J.H.; TYLER, J.W. et al. Advances in the therapy for mastitis. Vet. Clin. N. Am.: Food Anim. Pract., v.9, p.499-513, 1993.

MACEDO, M.L.A.P.; CARTAXO, R.S.; ALMEIDA, T.C.C. et al. Mecanismos de resistência e detecção das betalactamases. Cienc. Biol. Saúde, v.7, p.59-63, 2005.

MEDEIROS, E.S.; MOTA, R.A.; SANTOS, M.V. et al. Perfil de sensibilidade microbiana in vitro de linhagens de Staphylococcus spp. isoladas de vacas com mastite subclínica. Pesq. Vet. Bras., v.29, p.569-574, 2009.

METHODS for dilution antimicrobial susceptibility tests for bacteria that grow aerobically: approved standard - 6.ed. Wayne, Pa: NCCLS, 2003 (document M7-A6).

PORTO, A.L.F.; CAMPOS-TAKAKI, G.M.; LIMA FILHO, J.L. Effects of culture conditions on protease production by Streptomyces clavuligerus growing soy bean flour medium. Appl. Biochem. Biotech., v.60, p.115-122, 1996.

PRIDHAM, T.G.; ADERSON, P.; FOLEY, C. et al. Selection of media for maintenance and taxonomic study of Streptomyces. Antibiot. Ann., p.947-953, 1956-1957. 
RIBEIRO, M.E.R.; PETRINI, L.A.; AITA, M.F. et al. Relação entre mastite clinica, subclinica infecciosa e não infecciosa em unidades de produção leiteiras na região sul do Rio Grande do Sul. Rev. Bras. Agric., v.9, p.287-290, 2003.

SALASIA, S.I.O. Comparative studies on phenoand genotypic properties of Staphylococcus aureus isolated from bovine subclinical mastitis in central Java in Indonésia and Hesse in Germany. J. Vet. Sci., v.5, p.103-109, 2004.

THAKUR, D.; YADAY, B.K.G.; BORA, T.C. Isolation and screening of Streptomyces in soil of protected forest areas from the states of Assam and Tripura, India, for antimicrobial metabolites. J. Mycol. Med., v.17, p.242-249, 2007.

TOZZETI, D.S.; BATAIER, M.B.N.; ALMEIDA, L.R. Prevenção, controle e tratamento das mastites bovinas - Revisão de literatura. Rev. Cien. Elet. Med. Vet., v.10, 2008.
TRESOLDI, A.T.; BARISON, E.M.; PEREIRA, R.M. et al. Risck factors associeted witch the acquisition of multiresistant bacteria in pedriatic nursery. J. Pediatr., v.4, p.275-286, 2000.

YU, T.W.; SHEN, Y.; DOI-KATAYAMA, Y. et al. Direct evidence that the rifamycin polyketide synthase assembles polyketides chains processively. Proc. Natl. Acad. Sci. USA, v.96, p.9051-9056, 1999

ZAFALON, L.F.; LANGONI, H.; BENVENUTTO, F. et al. Aspectos epidemiológicos da mastite bovina causada por Staphylococcus aureus. Vet. Zootec., v.15, p.5665, 2008. 